\title{
Cohomological divisibility and point count divisibility
}

\author{
Hélène Esnault and Nicholas M. Katz
}

\begin{abstract}
Let $X \subset \mathbb{P}^{n}$ be a closed scheme defined by $r$ homogeneous equations of degrees $d_{1} \geqslant d_{2} \geqslant$ $\cdots \geqslant d_{r}$ over the finite field $\mathbb{F}_{q}$, with complement $U:=\mathbb{P}^{n} \backslash X$. Let $\kappa$ be the maximum of 0 and the integral part of the rational number $\left(n-d_{2}-\cdots-d_{r}\right) / d_{1}$. We show that the eigenvalues of the geometric Frobenius endomorphism acting on the $\ell$-adic cohomology $H_{\mathrm{c}}^{i}\left(U \times_{\mathbb{F}_{q}} \overline{\mathbb{F}_{q}}, \mathbb{Q}_{\ell}\right)$ with compact supports are divisible by $q^{\kappa}$ as algebraic integers.
\end{abstract}

\section{Introduction}

Nearly seventy years have passed since the theorem of Chevalley-Warning [Che36, War36]: over a finite field $k=\mathbb{F}_{q}$ of characteristic $p$, if $f \in k\left[X_{1}, \ldots, X_{n}\right]$ is a polynomial in $n \geqslant 1$ variables of degree $d<n$, then the number of solutions in $\mathbb{A}^{n}(k)$ of $f=0$ is divisible by $p$. For $d \geqslant 1$, Ax [Ax64] improved this to divisibility by $q^{\mu}$, where $\mu$ is the least non-negative integer that is $\geqslant(n-d) / d$. Thus

$$
\mu=\max \left(0, \text { Ceiling }\left(\frac{n-d}{d}\right)\right) .
$$

In this form, the result remains true, if trivially so, without the assumption that $n>d$. Ax also noted that, by an elementary inclusion-exclusion argument, his result implied that if one had $r \geqslant 1$ polynomials $f_{i} \in k\left[X_{1}, \ldots, X_{n}\right]$, with $f_{i}$ of degree $d_{i} \geqslant 1$, then the number of solutions in $\mathbb{A}^{n}(k)$ of the system of equations $f_{1}=\cdots=f_{r}=0$ is divisible by $q^{\lambda}$, where $\lambda$ is the least non-negative integer which is $\geqslant\left(n-\sum_{i} d_{i}\right) / \sum_{i} d_{i}$, i.e.

$$
\lambda=\max \left(0, \text { Ceiling }\left(\frac{n-\sum_{i} d_{i}}{\sum_{i} d_{i}}\right)\right) .
$$

Katz [Kat71] improved this to divisibility by $q^{\mu}$, where $\mu$ is the least non-negative integer which is $\geqslant\left(n-\sum_{i} d_{i}\right) / \max _{i} d_{i}$, i.e.

$$
\mu:=\mu\left(n ; d_{1}, \ldots, d_{r}\right):=\max \left(0, \text { Ceiling }\left(\frac{n-\sum_{i} d_{i}}{\max _{i} d_{i}}\right)\right) .
$$

An elementary proof of this result was given by Wan [Wan89]. Adolphson and Sperber [AS87] gave a divisibility estimate for additive character sums which, they showed, included this result. Wan [Wan95] gave a divisibility estimate for multiplicative character sums which, he showed, included this result as well.

Del Angel noted in [dAn94] that, if one reverses the inclusion-exclusion argument which Ax used to pass from a result for one polynomial to a result for several polynomials, then the estimate (1.3) for several polynomials gives the following result for one polynomial which is reducible: if one has $r \geqslant 1$ polynomials $f_{i} \in k\left[X_{1}, \ldots, X_{n}\right]$, with $f_{i}$ of degree $d_{i} \geqslant 1$, then the number of solutions

Received 12 May 2003, accepted in final form 21 January 2004, published online 1 December 2004. 2000 Mathematics Subject Classification 14G10, $14 \mathrm{G} 15$.

Keywords: Ax-Katz theorem, eigenvalues of Frobenius, rational points over finite fields.

This journal is (C) Foundation Compositio Mathematica 2005. 


\section{H. Esnault And N. M. Katz}

in $\mathbb{A}^{n}(k)$ of the single equation $f_{1} \cdots f_{r}=0$ is divisible by $q^{\mu}$, for $\mu=q^{\mu\left(n ; d_{1}, \ldots, d_{r}\right)}$ as above. If one replaces $f_{1} \cdots f_{r}$ by any product $f_{1}^{a_{1}} \cdots f_{r}^{a_{r}}$ with all $a_{i} \geqslant 1$, one has the same solutions in $\mathbb{A}^{n}(k)$, so the same divisibility. Notice that if one omits some of the factors in $f_{1}^{a_{1}} \cdots f_{r}^{a_{r}}$, i.e. if one allows some $a_{i}$ to be zero, then the number of solutions of $f_{1}^{a_{1}} \cdots f_{r}^{a_{r}}=0$ in $\mathbb{A}^{n}(k)$ remains divisible by the same $q^{\mu}$ [simply because if one removes some of the $f_{i}$ from consideration, the numerator $n-\sum_{i} d_{i}$ of $\mu$ increases, and its denominator $\max _{i} d_{i}$ decreases].

With this result in hand, del Angel did inclusion-exclusion à $l a \mathrm{Ax}$ and got the following result. Suppose one has a list of $r \geqslant 1$ polynomials $f_{i} \in k\left[X_{1}, \ldots, X_{n}\right]$, with $f_{i}$ of degree $d_{i} \geqslant 1$. Take any

$R \geqslant 1$, and any list of $R \geqslant 1$ polynomials $g_{j} \in k\left[X_{1}, \ldots, X_{n}\right]$, such that $g_{j}$ is a product $g_{j}=\prod_{i} f_{i}^{a_{i, j}}$, with all $a_{i, j} \geqslant 0$. Then the equations $g_{1}=\cdots=g_{R}=0$ define a closed subscheme $X \subset \mathbb{A}^{n}$, whose number of $k$-points is divisible by $q^{\mu\left(n ; d_{1}, \ldots, d_{r}\right)}$.

There is an obvious projective version of these affine results. If the $f_{i}$ are all homogeneous, then all the $g_{j}$ are homogeneous, and the same equations $g_{1}=\cdots=g_{R}=0$ also define a closed subscheme $X^{\text {proj }} \subset \mathbb{P}^{n-1}$ whose affine cone is the $X$ above. The number of $\mathbb{F}_{q}$-rational points of $U:=\mathbb{P}^{n-1} \backslash X^{\text {proj }}$ is easily seen to be divisible by $q^{\mu\left(n ; d_{1}, \ldots, d_{r}\right)}$.

The common feature of these results is that one has a separated $k$-scheme $X / k$ of finite type, and an integer $\kappa \geqslant 1$, such that for each finite extension $\mathbb{F}_{q^{\nu}}$ of $\mathbb{F}_{q},\left|X\left(\mathbb{F}_{q^{\nu}}\right)\right|$ is divisible by $q^{\nu \cdot \kappa}$. Some years ago, Deligne posed the problem of characterizing those situations $(X / k, \kappa)$ in which the divisibilities of numbers of points result from corresponding divisibilities of all the eigenvalues of Frobenius on all the compact $\ell$-adic cohomology groups, $\ell$ any prime other than $p$, of the variety $X / k$ in question. As explained by Ax [Ax64], such divisibilities of numbers of points imply (and indeed are equivalent to) the corresponding divisibility of all reciprocal zeros and poles of the zeta function. But for any given $\ell \neq p$, the $\ell$-adic cohomological expression of the zeta function may well have cancellation: the problem is that there might be pairs of Frobenius eigenvalues on different $\ell$-adic groups which cancel, but which both have insufficient divisibility. An example of such an $X$ is the disjoint union of a point of degree one over $\mathbb{F}_{q}$ with $\mathbb{G}_{m} \times \mathbb{P}^{n-1}$. Then it has exactly $q^{n}$ rational points while Frobenius acts trivially on the zeroth and the first cohomology with compact supports.

In this paper, we show that, if $(X / k, \kappa)$ arises as an instance of the point count divisibility theorems [Kat71] explained above, then in fact all its Frobenius eigenvalues have the corresponding divisibility.

We should emphasize that in proving the asserted divisibilities of Frobenius eigenvalues, we are not giving a new proof of the above-cited divisibilities of point counts. Rather, those point count divisibilities are an essential starting point of our proof; see (3.6). (For another approach to these questions, see [Esn03], which treats the case of projective complete intersections. For a discussion of a motivic approach, see the survey [BE02].) In $\S 5.3$, we explain how our methods can be used to sharpen known results on the analogous question of Hodge type of complex projective varieties defined by equations of low degree, cf. [DD90, Esn90, ENS92, dAn94, EW03].

\section{Statement of results: the affine case}

We fix a finite field $k=\mathbb{F}_{q}$ of characteristic $p$ and a prime number $\ell \neq p$. For a separated scheme $X / k$ of finite type, we abbreviate

$$
H^{i}(X):=H^{i}\left(X \times_{k} \bar{k}, \mathbb{Q}_{\ell}\right), \quad H_{\mathrm{c}}^{i}(X):=H_{\mathrm{c}}^{i}\left(X \times_{k} \bar{k}, \mathbb{Q}_{\ell}\right) .
$$




\section{Cohomological Divisibility}

We denote by $F=F_{k}$ the geometric Frobenius endomorphism of these cohomology groups. By the Grothendieck-Lefschetz trace formula [Gro65],

$$
\begin{aligned}
|X(k)| & =\sum_{i}(-1)^{i} \operatorname{Trace}\left(F ; H_{\mathrm{c}}^{i}(X)\right), \\
\left|X\left(k_{\nu}\right)\right| & =\sum_{i}(-1)^{i} \operatorname{Trace}\left(F^{\nu} ; H_{\mathrm{c}}^{i}(X)\right),
\end{aligned}
$$

for $k_{\nu}=\mathbb{F}_{q^{\nu}}$. Recall [Del73b, Corollaire 5.5.3], that all the eigenvalues of $F$ acting on $H_{\mathrm{c}}^{i}(X)$ are algebraic integers, i.e. elements of $\overline{\mathbb{Q}_{\ell}}$ which are integral over $\mathbb{Z}$.

Theorem 2.1. Suppose one has a list of $r \geqslant 1$ polynomials $f_{i} \in k\left[X_{1}, \ldots, X_{n}\right]$, with $f_{i}$ of degree $d_{i} \geqslant 1$. Take any integer $R \geqslant 1$, and any list of $R \geqslant 1$ polynomials $g_{j} \in k\left[X_{1}, \ldots, X_{n}\right]$, such that each $g_{j}$ is a product $g_{j}=\prod_{i} f_{i}^{a_{i, j}}$, with all $a_{i, j} \geqslant 0$. Denote by $X / k$ the closed subscheme of $\mathbb{A}^{n}(k)$ defined by $g_{1}=\cdots=g_{R}=0$. Then every eigenvalue of $F$ on every compact cohomology group $H_{\mathrm{c}}^{i}(X)$ is divisible as an algebraic integer by $q^{\mu\left(n ; d_{1}, \ldots, d_{r}\right)}$.

From the excision sequence

$$
\cdots \rightarrow H_{\mathrm{c}}^{i}\left(\mathbb{A}^{n} \backslash X\right) \rightarrow H_{\mathrm{c}}^{i}\left(\mathbb{A}^{n}\right) \rightarrow H_{\mathrm{c}}^{i}(X) \rightarrow \cdots,
$$

and the well-known cohomological structure of $\mathbb{A}^{n}$, namely

$$
H_{\mathrm{c}}^{i}\left(\mathbb{A}^{n}\right)=0 \text { for } i \neq 2 n, \quad H_{\mathrm{c}}^{2 n}\left(\mathbb{A}^{n}\right)=\mathbb{Q}_{\ell}(-n),
$$

where $\mathbb{Q}_{\ell}(-n)$ is the one-dimensional vector space over $\mathbb{Q}_{\ell}$ on which $F$ acts as $q^{n}$, one sees that Theorem 2.1 is equivalent to the following theorem.

THEOREM 2.2. Under the hypotheses of Theorem 2.1, every eigenvalue of $F$ on every compact cohomology group $H_{\mathrm{c}}^{i}\left(\mathbb{A}^{n} \backslash X\right)$ is divisible as an algebraic integer by $q^{\mu\left(n ; d_{1}, \ldots, d_{r}\right)}$.

Theorem 2.2 itself results from the following slightly sharper result, suggested by [Del73b, Corollaire 5.3.3, (ii)], and by [Wan02] and [EW03]. For each integer $j \geqslant 0$, let us define

$$
\mu_{j}\left(n ; d_{1}, \ldots, d_{r}\right):=j+\mu\left(n-j ; d_{1}, \ldots, d_{r}\right) .
$$

Thus

$$
\mu_{0}\left(n ; d_{1}, \ldots, d_{r}\right)=\mu\left(n ; d_{1}, \ldots, d_{r}\right),
$$

and we have the obvious inequalities

$$
\mu_{j+1}\left(n ; d_{1}, \ldots, d_{r}\right) \geqslant \mu_{j}\left(n ; d_{1}, \ldots, d_{r}\right) .
$$

TheOREm 2.3. Under the hypotheses of Theorem 2.1, we have the following results:

1) Every eigenvalue of $F$ on every group $H_{\mathrm{c}}^{i}\left(\mathbb{A}^{n} \backslash X\right)$ is divisible as an algebraic integer by $q^{\mu\left(n ; d_{1}, \ldots, d_{r}\right)}$.

2) For each $j \geqslant 1$, every eigenvalue of $F$ on $H_{\mathrm{c}}^{n+j}\left(\mathbb{A}^{n} \backslash X\right)$ is divisible as an algebraic integer by $q^{\mu_{j}\left(n ; d_{1}, \ldots, d_{r}\right)}$. 


\section{H. Esnault And N. M. Katz}

\section{The proof of Theorem 2.3}

We first reduce to the case $R=1$. For $j$, with $1 \leqslant j \leqslant R$, we denote by $G_{j} \subset \mathbb{A}^{n}$ the hypersurface in $\mathbb{A}^{n}$ defined by $g_{j}=0$. Then

$$
\begin{gathered}
X=\bigcap_{j=1}^{R} G_{j}, \quad \text { hence } \\
\mathbb{A}^{n} \backslash X=\bigcup_{j=1}^{R} U_{j}, \quad \text { with } U_{j}=\mathbb{A}^{n} \backslash G_{j} .
\end{gathered}
$$

One considers the Mayer-Vietoris spectral sequence for compact cohomology (see [Ver66] and [Del73a, 6.2.10.3]),

$$
E_{1}^{-a, b}=\bigoplus_{1 \leqslant j_{1}<j_{2}<\cdots<j_{a+1} \leqslant R} H_{\mathrm{c}}^{b}\left(\bigcap_{i=1}^{a+1} U_{j_{i}}\right) \Rightarrow H_{\mathrm{c}}^{b-a}\left(\mathbb{A}^{n} \backslash X\right),
$$

which is the cohomological incarnation of inclusion-exclusion. Thus every eigenvalue of $F$ on a given group $H_{\mathrm{c}}^{b}\left(\mathbb{A}^{n} \backslash X\right)$ is an eigenvalue of $F$ on some group $H_{\mathrm{c}}^{b+a}\left(\bigcap_{i=1}^{a+1} U_{j_{i}}\right)$ for some $a \geqslant 0$. So it suffices to show the following two statements:

1) For every $b \geqslant 0$ and every $a \geqslant 0$, every eigenvalue of $F$ on every group $H_{\mathrm{c}}^{b}\left(\bigcap_{i=1}^{a+1} U_{j_{i}}\right)$ is divisible as an algebraic integer by $q^{\mu\left(n ; d_{1}, \ldots, d_{r}\right)}$.

2) For every $j \geqslant 1$ and every $a \geqslant 0$, every eigenvalue of $F$ on every group $H_{\mathrm{c}}^{n+j}\left(\bigcap_{i=1}^{a+1} U_{j_{i}}\right)$ is divisible as an algebraic integer by $q^{\mu_{j}\left(n ; d_{1}, \ldots, d_{r}\right)}$.

But an $(a+1)$-fold intersection $\bigcap_{i=1}^{a+1} U_{j_{i}}$ is the complement of the hypersurface of the equation $\prod_{i=1}^{a+1} g_{j_{i}}=0$, which falls under the case $R=1$ of Theorem 2.3. So it suffices to prove Theorem 2.3 for $R=1$. We use the weak Lefschetz theorem and induction on $n$, the number of variables. For $n=1$, we have $\mu\left(1 ; d_{1}, \ldots, d_{r}\right)=0$ (because $\sum_{i} d_{i} \geqslant 1$ ), so assertion 1 of the theorem is an (easy) instance of Deligne's integrality theorem [Del73b, Corollaire 5.5.3]. Assertion 2 of the theorem holds for $n=1$ because the only possibly non-zero group $H_{\mathrm{c}}^{1+j}\left(\mathbb{A}^{1} \backslash X\right)$ with $j \geqslant 1$ is $H_{\mathrm{c}}^{2}\left(\mathbb{A}^{1} \backslash X\right)$, which is $\mathbb{Q}_{\ell}(-1)$. So suppose the theorem universally true for $n-1$, in the case $R=1$.

Whatever the non-empty hypersurface $G \subset \mathbb{A}^{n}, \mathbb{A}^{n} \backslash G$ is smooth and geometrically connected of dimension $n$, so Poincaré duality tells us that the cup product

$$
H_{\mathrm{c}}^{i}\left(\mathbb{A}^{n} \backslash G\right) \times H^{2 n-i}\left(\mathbb{A}^{n} \backslash G\right) \rightarrow H_{\mathrm{c}}^{2 n}\left(\mathbb{A}^{n} \backslash G\right) \cong \mathbb{Q}_{\ell}(-n)
$$

is a perfect pairing of $F$-modules. Since $\mathbb{A}^{n} \backslash G$ is affine of dimension $n$, the affine Lefschetz theorem tells us that

$$
H^{i}\left(\mathbb{A}^{n} \backslash G\right)=0 \text { for } i>n .
$$

So by duality we have

$$
H_{\mathrm{c}}^{i}\left(\mathbb{A}^{n} \backslash G\right)=0 \text { for } i<n .
$$

We now take $G$ defined by $\prod_{i} f_{i}^{a_{i}}=0$.

We first prove assertion 1 of the theorem. As noted by Wan and exploited in [BEL04, Introduction], and in [Esn03], given the divisibility of

$$
\left|\left(\mathbb{A}^{n} \backslash G\right)\left(k_{\nu}\right)\right|=\sum_{i \geqslant n}(-1)^{i} \operatorname{Trace}\left(F^{\nu} ; H_{\mathrm{c}}^{i}\left(\mathbb{A}^{n} \backslash G\right)\right)
$$

by $q^{\nu \cdot \mu}$ for every $\nu \geqslant 1$, it suffices to prove that for all but a single value of $i$ we have the asserted divisibility of Frobenius eigenvalues on $H_{\mathrm{c}}^{i}\left(\mathbb{A}^{n} \backslash G\right)$. We choose $i=n$. So we need only show the divisibility by $q^{\mu\left(n ; d_{1}, \ldots, d_{r}\right)}$ of Frobenius eigenvalues on $H_{\mathrm{c}}^{j}\left(\mathbb{A}^{n} \backslash G\right)$ for $j>n$. 


\section{Cohomological Divisibility}

This divisibility is invariant under finite extension of the ground field; after a degree- $d$ extension of the ground field, both eigenvalues and $q$ are replaced by their $d$ th power. For a sufficiently general affine linear hyperplane $A \subset \mathbb{A}^{n}$ defined by an equation of the form

$$
A: \sum_{i=1}^{n} \alpha_{i} X_{i}+\beta_{i}=0
$$

with coefficients in a finite extension of $k$ (which, by extending $k$, we may assume to be $k$ itself), the restriction maps on ordinary cohomology

$$
H^{i}\left(\mathbb{A}^{n} \backslash G\right) \rightarrow H^{i}(A \backslash A \cap G)
$$

are injective for $i<n$. This is an instance of the weak Lefschetz theorem (see [Kat93, 3.4.1], applied to $V=\mathbb{A}^{n} \backslash G$, with $\pi$ the inclusion and $f$ the function 0 ). The Poincaré dual of this injection is a surjection

$$
H_{\mathrm{c}}^{i-2}(A \backslash A \cap G)(-1) \rightarrow H_{\mathrm{c}}^{i}\left(\mathbb{A}^{n} \backslash G\right)
$$

for $i>n$. For $A$ general, each $\left.f_{i}\right|_{A}$ will be a polynomial of the same degree $d_{i}$. So by induction on $n$, we know that every eigenvalue of $F$ on every group $H_{\mathrm{c}}^{i-2}(A \backslash A \cap G)$ is divisible by $q^{\mu\left(n-1 ; d_{1}, \ldots, d_{r}\right)}$. So every eigenvalue of $F$ on every group $H_{\mathrm{c}}^{i-2}(A \backslash A \cap G)(-1)$ is divisible by $q^{\mu\left(n-1 ; d_{1}, \ldots, d_{r}\right)+1}$. But we have the inequality

$$
\mu\left(n-1 ; d_{1}, \ldots, d_{r}\right)+1=\mu_{1}\left(n ; d_{1}, \ldots, d_{r}\right) \geqslant \mu\left(n ; d_{1}, \ldots, d_{r}\right) .
$$

This concludes the proof of assertion 1 of Theorem 2.3.

This same induction proves assertion 2 of Theorem 2.3. Indeed, for any integer $j \geqslant 1$, the induction shows that, for $i=n+j$, every eigenvalue of $F$ on $H_{\mathrm{c}}^{n+j}\left(\mathbb{A}^{n} \backslash G\right)$ is also an eigenvalue of $F$ on

$$
H^{(n-1)+(j-1)}(A \backslash G \cap A)(-1) .
$$

By induction, every eigenvalue of $F$ on $(3.11)$ is divisible by $q^{1+\mu_{j-1}\left(n ; d_{1}, \ldots, d_{r}\right)}$. Also by definition we have

$$
1+\mu_{j-1}\left(n ; d_{1}, \ldots, d_{r}\right)=\mu_{j}\left(n ; d_{1}, \ldots, d_{r}\right) .
$$

This concludes the proof of Theorem 2.3.

\section{The projective case}

Here is the projective version. Suppose now that the $f_{i}$ are all homogeneous forms. Then each $g_{j}$ is again homogeneous, and the closed $X / k$ in $\mathbb{A}^{n}$ defined by $g_{1}=\cdots=g_{R}=0$ is the affine cone over

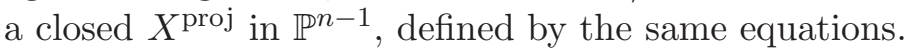

Theorem 4.1. Under the hypotheses of Theorem 2.1, suppose in addition that all the $f_{i}$ are homogeneous. Then we have the following results:

1) Every eigenvalue of $F$ on every group $H_{\mathrm{c}}^{i}\left(\mathbb{P}^{n-1} \backslash X^{\mathrm{proj}}\right)$ is divisible as an algebraic integer by $q^{\mu\left(n ; d_{1}, \ldots, d_{r}\right)}$.

2) For each $j \geqslant 1$, every eigenvalue of $F$ on $H_{\mathrm{c}}^{n+j}\left(\mathbb{P}^{n-1} \backslash X^{\mathrm{proj}}\right)$ is divisible as an algebraic integer by $q^{\mu_{j}\left(n ; d_{1}, \ldots, d_{r}\right)}$.

Proof. To prove the theorem, repeat the proof of Theorem 2.3 essentially verbatim. First reduce to the case $R=1$ by the same spectral sequence argument. Then do induction on $n$. Again, the case

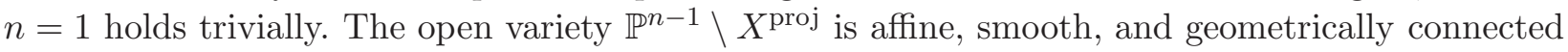
of dimension $n-1$. The appropriate reference for the weak Lefschetz theorem in this context 


\section{H. Esnault And N. M. Katz}

is [Kat93, Appendix (d'après Deligne), A5], applied with its $X:=\mathbb{P}^{n-1} \backslash X^{\text {proj }}$, and $f$ the inclusion into $\mathbb{P}^{n-1}$.

\section{Concluding remarks}

\subsection{Concordance}

In other work on this subject, e.g. in [Esn90, ENS92, EW03], one sees a different but equivalent expression of the divisibility, in terms of the floor or integral part $[x]$ of a real number $x$. One renumbers so that $d_{1} \geqslant d_{2} \geqslant \cdots \geqslant d_{r}$, and then one defines

$$
\kappa\left(n ; d_{1}, \ldots, d_{r}\right):=\max \left(0,\left[\frac{n-d_{2}-\cdots-d_{r}}{d_{1}}\right]\right) .
$$

This $\kappa$ is related to $\mu$ by the identity

$$
\kappa\left(n ; d_{1}, \ldots, d_{r}\right)=\mu\left(n+1 ; d_{1}, \ldots, d_{r}\right),
$$

whose verification we leave to the reader.

\subsection{Axiomatization}

The arguments we give here could easily be axiomatized, and then applied to any pair of cohomology theories $\left(H^{\star}, H_{\mathrm{c}}^{\star}\right)$, defined on smooth separated $\bar{k}$-schemes of finite type, with values in finitedimensional vector spaces over a field $K$ of characteristic zero, which have the following properties:

i) zeta function formula for $H_{\mathrm{c}}^{\star}$,

ii) Poincaré duality between $H^{\star}$ and $H_{\mathrm{c}}^{\star}$,

iii) affine Lefschetz and weak Lefschetz theorems for $H^{\star}$,

iv) Mayer-Vietoris spectral sequence of an open covering for $H_{\mathrm{c}}^{\star}$.

An initial run through our arguments with $\mu=0$ shows that all Frobenius eigenvalues on $H_{\mathrm{c}}^{\star}$ in any such theory are algebraic integers. Berthelot's rigid cohomology and rigid cohomology with compact supports are conjecturally such a pair of theories. At present, properties i, ii and iv are proven in [ELS93], [Ber97b] and [Ber97a] respectively. Only property iii remains open. Once iii has been proven, then Theorems 2.3 and 4.1 will hold for the eigenvalues of Frobenius on compact rigid cohomology, with divisibility by $q^{\mu}$ as algebraic integers and hence with slopes of Frobenius $\geqslant \mu$.

\subsection{Application to Hodge type}

Deligne has also formulated a Hodge theoretic version of the problem considered here. Given a nonempty separated $\mathbb{C}$-scheme $X / \mathbb{C}$ of finite type, recall that its compact support Hodge type $\alpha(X / \mathbb{C})$ is the largest integer $\alpha$ such that the Hodge filtration Fil, for the mixed Hodge structure on $H_{\mathrm{c}}^{\star}(X)$, satisfies

$$
\operatorname{Fil}^{\alpha} H_{\mathrm{c}}^{\star}(X)=H_{\mathrm{c}}^{\star}(X) \text {. }
$$

We can also 'spread out' $X / \mathbb{C}$ to a separated scheme $\mathcal{X} / A$ of finite type, with $A$ a subring of $\mathbb{C}$ which is finitely generated as a $\mathbb{Z}$-algebra. For each such spreading out $\mathcal{X} / A$, we define $\beta(\mathcal{X} / A)$ to be the largest integer $\beta$ with the following property: for every finite field $\mathbb{F}_{q}$, and for every ring homomorphism $\phi: A \rightarrow \mathbb{F}_{q}$, the number of points on the scheme $\mathcal{X}_{\phi} / \mathbb{F}_{q}$ obtained from $\mathcal{X} / A$ by the extension of scalars $\phi$ is divisible by $q^{\beta}$. We then define $\beta(X / \mathbb{C})$ to be the maximum of $\beta(\mathcal{X} / A)$ for all spreadings out of $X / \mathbb{C}$. One sees easily that $\beta(X / \mathbb{C}) \leqslant \operatorname{dim}(X)$. The problem is to characterize those $X / \mathbb{C}$ for which $\alpha(X / \mathbb{C})=\beta(X / \mathbb{C})$. (Presumably, when this equality holds, it holds because $\alpha(X / \mathbb{C})$ and $\beta(X / \mathbb{C})$ are both equal to some intrinsic 'motivic' invariant $\gamma(X / \mathbb{C})$ cf. [Gro68, sections 9-10], for Grothendieck's discussion of this sort of question.) In general, not much is known. There are 


\section{CoHomological Divisibility}

simple cases where this equality fails, e.g. the disjoint union of a rational point with the product $\mathbb{G}_{m} \times \mathbb{P}^{n-1}$. But in cases where the divisibility theorems of Ax et al. give an estimate $\beta(X / \mathbb{C}) \geqslant \kappa$, one knows that $\alpha(X / \mathbb{C}) \geqslant \kappa$ (cf. [DD90, ENS92, dAn94, EW03]).

The methods we use here allow us to sharpen these last results. Given the known results on the Hodge type of hypersurfaces as input [DD90, Esn90], the arguments proving Theorems 2.3 and 4.1 also prove their analogues over $\mathbb{C}$, with eigenvalue divisibility replaced by Hodge type. We should emphasize that we are not giving a new proof of the known results on the Hodge type of hypersurfaces, but rather using them as a starting point.

Consider first the projective case. The first assertion of the Hodge analogue of Theorem 4.1 is the result [DD90, Esn90, ENS92, dAn94]. To prove the second assertion, reduce to the $R=1$ case by the spectral sequence, and then use induction and the weak Lefschetz theorem exactly as in the finite field case. Consider now the affine case, i.e. the Hodge analogue of Theorem 2.3. The spectral sequence reduces us to the case $R=1$. Once we know the first assertion in the case $R=1$, we get the second assertion in that case by using induction and the affine weak Lefschetz theorem, exactly as in the finite field case. To prove the first assertion in the $R=1$ case, we reduce it to the projective case. For this reduction, for each $f_{i} \in \mathbb{C}\left[X_{1}, \ldots, X_{n}\right]$, with $f_{i}$ of degree $d_{i} \geqslant 1$, denote by $F_{i} \in \mathbb{C}\left[X_{0}, \ldots, X_{n}\right]$ the homogeneous form of the same degree $d_{i}$ such that

$$
f_{i}\left(X_{1}, \ldots, X_{n}\right)=F_{i}\left(1, X_{1}, \ldots, X_{n}\right),
$$

and denote by $F_{i, 0} \in \mathbb{C}\left[X_{1}, \ldots, X_{n}\right]$ the leading form of $f_{i}$, i.e.

$$
F_{i, 0}\left(X_{1}, \ldots, X_{n}\right)=F_{i}\left(0, X_{1}, \ldots, X_{n}\right) .
$$

Given $G$ the hypersurface in $\mathbb{A}^{n}$ defined by $\prod_{i} f_{i}^{a_{i}}=0$, denote by $G^{\text {proj }}$ the hypersurface in $\mathbb{P}^{n}$ defined by $\prod_{i} F_{i}^{a_{i}}=0$, and denote by $G_{0}^{\text {proj }}$ the hypersurface in $\mathbb{P}^{n-1}$ defined by $\prod_{i} F_{i, 0}^{a_{i}}=0$. Then we have an excision sequence

$$
\cdots \rightarrow H_{\mathrm{c}}^{i-1}\left(\mathbb{P}^{n-1} \backslash G_{0}^{\mathrm{proj}}\right) \rightarrow H_{\mathrm{c}}^{i}\left(\mathbb{A}^{n} \backslash G\right) \rightarrow H_{\mathrm{c}}^{i}\left(\mathbb{P}^{n} \backslash G^{\text {proj }}\right) \rightarrow \cdots .
$$

But $H_{\mathrm{c}}^{\star}\left(\mathbb{P}^{n-1} \backslash G_{0}^{\text {proj }}\right)$ has Hodge type at least $\mu\left(n ; d_{1}, \ldots, d_{r}\right)$, and $H_{\mathrm{c}}^{\star}\left(\mathbb{P}^{n} \backslash G^{\text {proj }}\right)$ has Hodge type at least

$$
\mu\left(n+1 ; d_{1}, \ldots, d_{r}\right) \geqslant \mu\left(n ; d_{1}, \ldots, d_{r}\right)
$$

Thus $H_{\mathrm{c}}^{\star}\left(\mathbb{A}^{n} \backslash G\right)$ has Hodge type at least $\mu\left(n ; d_{1}, \ldots, d_{r}\right)$, as required.

\section{ACKNOWLEDGEMENT}

We warmly thank Spencer Bloch for his interest in and incisive comments on the work leading up to this paper.

\section{REFERENCES}

AS87 A. Adolphson and S. Sperber, p-adic estimates for exponential sums and the theorem of ChevalleyWarning, Ann. Sci. École Norm. Sup. (4) 20 (1987), 545-556.

Ax64 J. Ax, Zeroes of polynomials over finite fields, Amer. J. Math. 86 (1964), 255-261.

Ber97a P. Berthelot, Finitude et pureté cohomologique en cohomologie rigide, Invent. Math. 128 (1997), 329-377.

Ber97b P. Berthelot, Dualité de Poincaré et formule de Künneth en cohomologie rigide, C. R. Acad. Sci. Paris Sér. I Math. 325 (1997), 493-498.

BE02 S. Bloch and H. Esnault, Congruences for the number of rational points, Hodge type and motivic conjectures for Fano varieties, Cubo Mat. Educ. 5 (2003), 251-262. 


\section{Cohomological DivisibiLity}

BEL04 S. Bloch, H. Esnault and M. Levine, Decomposition of the diagonal and eigenvalues of Frobenius for Fano hypersurfaces, Amer. J. Math., to appear.

Che36 C. Chevalley, Démonstration d'une hypothèse de M. Artin, Abh. Math. Sem. Univ. Hamburg 11 (1936), 73-75.

dAn94 P.-L. del Angel, A remark on the Hodge type of projective varieties of low degree, J. reine angew. Math. 449 (1994), 173-177.

DD90 P. Deligne and A. Dimca, Filtrations de Hodge et par l'ordre du pôle pour les hypersurfaces singulières, Ann. Sci. École Norm. Sup. (4) 23 (1990), 645-656.

Del73a P. Deligne, Cohomologie à support propre, avec Appendice de B. Saint-Donat, exposé XVII, in SGA 4, tome 3, Lecture Notes in Mathematics, vol. 305 (Springer, Berlin, 1973), 250-480.

Del73b P. Deligne, Théorème d'intégralité, Appendix to N. Katz, Le niveau de la cohomologie des intersections complètes, exposé XXI, in SGA 7, Lecture Notes in Mathematics, vol. 340 (Springer, Berlin, 1973), 363-400.

ENS92 H. Esnault, M. Nori and V. Srinivas, Hodge type of projective varieties of low degree, Math. Ann. 293 (1992), 1-6.

Esn90 H. Esnault, Hodge type of subvarieties of $\mathbb{P}^{n}$ of small degrees, Math. Ann. 288 (1990), 549-551.

Esn03 H. Esnault, Eigenvalues of Frobenius acting on the $\ell$-adic cohomology of complete intersections of low degree, C. R. Math. Acad. Sci. Paris 337 (2003), 317-320.

EW03 H. Esnault and D. Wan, Hodge type of the exotic cohomology of complete intersections, C. R. Math. Acad. Sci. Paris 336 (2003), 153-157.

ELS93 J.-Y. Etesse and B. Le Stum, Fonctions L associées aux F-isocristaux surconvergents. I. Interprétation cohomologique, Math. Ann. 296 (1993), 557-576.

Gro65 A. Grothendieck, Formule de Lefschetz et rationalité des fonctions L, in Séminaire Bourbaki, 17ième année (1964/1965), vol. 9, exp. no. 279 (Soc. Mat. France, Paris, 1965), 41-55.

Gro68 A. Grothendieck, Le groupe de Brauer III: Exemples et compléments, in Dix exposés sur la cohomologie des schémas (North-Holland, Amsterdam and Masson, Paris, 1968).

Kat71 N. Katz, On a theorem of Ax, Amer. J. Math. 93 (1971), 485-499.

Kat93 N. Katz, Affine cohomological transforms, perversity, and monodromy, J. Amer. Math. Soc. 6 (1993), 149-222.

Ver66 J.-L. Verdier, A duality theorem in the étale cohomology of schemes, in Proc. Conf. Local Fields (Driebergen 1966) (Springer, Berlin, 1967), 184-198.

Wan89 D. Wan, An elementary proof of a theorem of Katz, Amer. J. Math. 11 (1989), 1-8.

Wan95 D. Wan, A Chevalley-Warning approach to p-adic estimates of character sums, Proc. Amer. Math. Soc. 123 (1995), 45-54.

Wan02 D. Wan, Poles of zeta functions of complete intersections, Chinese Ann. Math. 21B (2002), 187-200.

War36 E. Warning, Bemerkung zur vorstehenden Arbeit von Herrn Chevalley, Abh. Math. Sem. Univ. Hamburg 11 (1936), 76-83.

Hélène Esnault esnault@uni-essen.de

Universität Duisburg-Essen, FB6, Mathematik, 45117 Essen, Germany

Nicholas M. Katz nmk@math.princeton.edu

Princeton University, Department of Mathematics, Fine Hall, Princeton, NJ 08544-1000, USA 